\title{
Cytological Findings Suggesting Sexuality in Phytomonas davidi (Protozoa: Kinetoplastida)
}

\author{
Maria Auxiliadora de Sousa \\ Coleção de Tripanosomatídeos, Departamento de Protozoologia, Instituto Oswaldo Cruz, Av. Brasil 4365, \\ 21045-900 Rio de Janeiro, RJ, Brasil
}

On few occasions, Phytomonas davidi (McGhee \& Postell isolate) cultures in LIT (liver infusiontryptose) medium around $27^{\circ} \mathrm{C}$ presented, as seen in Giemsa-stained smears, a set of peculiar morphological features, among them being noticeable the pairs of apposed cells attached by their posterior ends, where occurred a stained line and/or a dilatation, usually bulb-like in shape; sometimes this dilatation could occupy one of the cells or hold both together. In some pairs, the nucleus of each parasite seemed migrating towards the other, entering into such dilatation; in others, both nuclei were inside it, sometimes in close proximity or seeming fused; peculiar chromatin arrangements involving both nuclei were occasionally observed. Several mono or binucleate round forms bearing one or two flagella, as well as flagellate slender cells without nucleus were concomitantly seen there. In some instances, an intriguing small stained body occurred beside a single large nucleus, either in pairs presenting the bulb-like structure or in round cells. These cytological findings seemed steps of a dynamic process suggesting sexuality, since in several of them nuclear interactions following fusion of two parasites appeared to occur.

Key words: trypanosomatid - Phytomonas davidi - morphology - sexuality

The increasing use of biochemistry and molecular biology techniques in the study of trypanosomatids has provided substantial amount of data suggesting sexuality, as in some Leishmania species (Maazoun et al. 1981, Le Blancq et al. 1983, Kelly et al. 1991, Cupolillo et al. 1992, Belli et al. 1994), or even proving it, as in Trypanosoma brucei (Jenni et al. 1986, Tait \& Turner 1990, Gibson \& Bailey 1994, Degen et al. 1995). Genetic recombination has also been reported in Crithidia fasciculata (Glassberg et al. 1985) following crosses between cloned populations differing in drug-resistance. Nevertheless, the morphological grounds of such findings have not yet been established and the first record of parasite fusion was given by Lanotte and Rioux (1990) on studying cultured promastigotes of $L$. infantum and $L$. tropica by videocinematography. Sousa (1994) also reported cytological events in cultures of Herpetomonas megaseliae strongly suggesting cell and nucleus fusion; similar phenomenon was also reported in L. major under unusual culture conditions (Sousa et al. 1993). The results presented herein were casually found in axenic cultures of Phytomonas davidi, isolate of McGhee and Postell

Fax: +55-21-290.9339

Received 2 September 1996

Accepted 9 December 1996
(1976), a trypanosomatid whose taxonomic position has been frequently discussed (Camargo et al. 1992), although its generic name remains in use (Wallace et al. 1992). The phenomenon described strongly suggests a sexual process, since morphological grounds for genetic exchange appear present, although at the level of this study this could not be demonstrated.

\section{MATERIALS AND METHODS}

The strain of $P$. davidi (ATCC 30287) used throughout this study was kindly supplied by Dr Erney P Camargo (São Paulo University, Brazil) on April 1991 and upon receipt was maintained in the Yaeger's liver infusion-tryptose (LIT) medium (Chiari \& Camargo 1984) containing penicillin $(200 \mathrm{U} / \mathrm{ml})$ and distributed in $4-5 \mathrm{ml}$ volumes into 16x150mm screwcap tubes. Cultures were kept at nearly $27^{\circ} \mathrm{C}$ and passed at 7-9 day intervals. Following the finding of unusual features in fresh preparations of 4-9 day old cultures, smears were prepared, fixed with methanol, treated with $\mathrm{HCl}$ $5 \mathrm{~N}$ (10-20 $\mathrm{min}$ ), subsequently washed and stained during $1 \mathrm{hr}$ with phosphate-buffered Giemsa solution (pH 7.2) (Carvalho 1973). These stained smears were studied by light microscopy at $\mathrm{X}$ 1,000 magnification and photographs were taken from the uncommon cytological findings; smears of a 6-day old culture were used to determine the percent of cells in the population presenting such features. Cultures of $P$. davidi maintained in our laboratory (LIT medium; around $27^{\circ} \mathrm{C}$ ) were ex- 
amined during more than five years, frequently at the first one and more sporadically thereafter. When the same peculiar features reappeared, Giemsa stained-smears were prepared from 3-day old cultures and a quantitative evaluation (\%) of morphological types was carried out. In this work all percents were determined by examining about 500 randomly selected forms.

\section{RESULTS}

After receipt, during three successive transfers in LIT medium, $P$. davidi cultures presented, as seen in Giemsa-stained smears, besides the typical promastigotes and longitudinally dividing forms, several unusual features, among them being noticeable the pairs of diametrically opposed cells joined by the posterior ends, where occurred a stained line (Figs 1-3, 19, arrowheads) and/or a dilatation, generally bulb-like in shape (Figs 3-6, $15,17,18,19,26,27,30)$. In pairs presenting the stained line, generally each cell could present its own nucleus (Figs 1, 3,19), but in some cases one of them had two nuclei (Fig. 2), sometimes close to each other, while the other cell was anucleate. The above-mentioned dilatation sometimes held both cells together (Figs 7-9, 20, 21, 24, arrowhead) or entirely occupied one cell of the pair (Figs $11,13,16,22,23,25,28)$; sometimes a clearer region was seen in such dilatation (Figs 4, arrowhead, 15, 16, 30). The nuclei of the joined cells sometimes appeared to be entering into the bulblike dilatation (Fig. 4), but frequently were seen inside or near it (Figs 5, 7, 8, 20,22). These nuclei could be in close proximity (Figs $5,7,8$ ) or apparently fused into a larger one (Figs 6, 9, 11, 21, 23), as well as could display peculiar chromatin arrangements (Figs 17, 18, 24, arrowhead, 29); sometimes one of them seemed to be degenerating (Fig. 30).

In these cultures there were also several mono or binucleate round forms bearing one or two flagella (Figs 10, 12, 14, 24), as well as several slender cells without nucleus, but retaining the kinetoplast and the flagellum (Fig. 12). Throughout this study, an intriguing small stained body was sometimes found near a single nucleus (usually large), either in pairs of apposed parasites (Figs 13, 16, 27,28 , arrows) or in round cells (Fig. 14, arrow). Three or more parasites joined by their posterior ends, and pairs of apposed cells presenting three nuclei (Figs 15, 25) were occasionally observed. Several cells with rounded posterior end or resembling a sucker and presenting one or two nuclei were found as well (Fig. 31). Giemsa-stained smears of a 6-day old culture revealed that $25.1 \%$ of the population presented the set of features here described, the pairs being $5.8 \%$.

In fresh preparations, the peculiar forms under study were also actively moving, being noteworthy the movement of pressure of a cell over the other observed in some parasite pairs presenting a well-developed bulb-like structure; an instantaneous of this movement is shown in Fig. 9.

The above-mentioned phenomenon was not seen in $P$. davidi cultures during more than five years, and without an evident cause reappeared on June 1996. The percents of the morphological types found on this occasion in 3-day old cultures are given in parentheses: pairs of apposed cells without (1.7) or with (5.6) the bulb-like dilatation; mono (7.4) or biflagellate (1.2) round cells; flagellate slender cells without nucleus (2.7); cells with rounded posterior end (4.1); sucker-like cells (2.5); longitudinally dividing cells (2.9); typical promastigotes (70.5); atypical or less frequent forms (1.4).

\section{DISCUSSION}

From the descriptions and illustrations presented in this paper, it seems clear that the peculiar pairs of cells found in $P$. davidi cultures have no similarity with longitudinally dividing parasites, furthermore being hardly understood as resulting from another type of division. Typically dividing trypanosomatids generally remain side-to-side and, when they are occasionally seen apposed each other before complete separation, neither a stained line nor any bulb-like structure are found between them, as here reported. However, several pairs of apposed cells described in the present paper resembled the pairs of promastigotes of Leishmania formed by fusion (Lanotte \& Rioux 1990), which began by contact of posterior ends of two diametrically opposed parasites, their adjacent membranes subsequently disappearing. Thus, the possibility is considered that the peculiar pairs seen in $P$. davidi also had been formed by fusion, the stained line between the cells probably being their contacting membranes at the beginning of the process; as such line was generally absent in pairs presenting the bulb-like dilatation, it is thought that complete fusion ensued.

The phenomenon here described also resembled the cell-to-cell interactions found in $L$. major and H. megaseliae (Sousa et al. 1993, Sousa 1994) which suggested sexuality, although having two remarkable peculiarities: the presence of the bulb-like structure joining two parasites, and the occurrence of mono or binucleate round cells bearing one or two flagella. On the other hand, no morphological feature of this phenomenon was similar to that one seen in $P$. davidi by França (1920) and questioned whether it was related to a sexual event. Furthermore, the findings reported herein in P. davidi also did not seem cellular aberrations (occasionally seen in any culture), mainly 


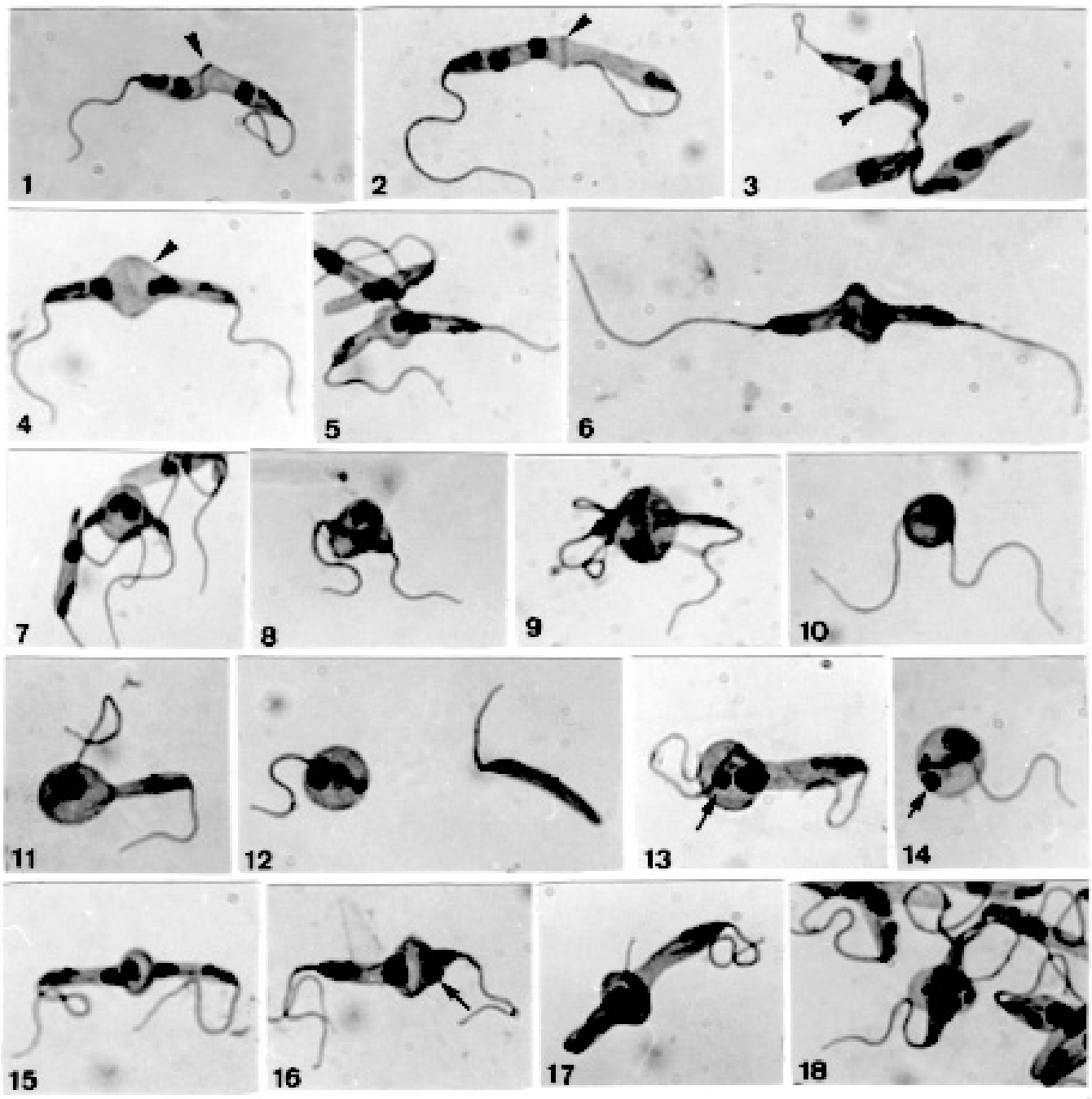

Unusual features in Phytomonas davidi cultured in LIT medium. Giemsa stain after $\mathrm{HCl}$ treatment. X 1,500. Fig. 1: promastigotes attached by the posterior ends, there occurring a stained line (arrowhead). Fig. 2: apposed cells having both nuclei inside one of them; note the stained line at the cellular junction (arrowhead). Fig. 3: note the enlargement and deep staining at the parasite junction (arrowhead). Fig. 4: bulb-like dilatation between apposed parasites, whose nuclei appear entering into it; note a clearer region there (arrowhead). Fig. 5: close proximity of nuclei in the apposed cells. Fig. 6: pair of parasites apparently with a fused nucleus. Figs 7-8: bulb-like structure holding two parasites, whose nuclei are near each other. Fig. 9: in this pair a pressure movement of a cell over the other is suggested; only one nucleus is seen. Fig. 10: biflagellate round cell apparently with a single nucleus. Fig. 11: bulb-like dilatation occupying one of the parasites, there occurring a large and apparently fused nucleus. Fig. 12: monoflagellate round cell with one nucleus (or two very close) and a slender cell without nucleus. Fig. 13: bulb-like dilatation occupying one of the parasites, where a small stained body (arrow) is seen beside a large nucleus. Fig. 14: monoflagellate round cell with a large nucleus and a small stained body (arrow). Fig. 15: pair of parasites with three nuclei; see the clear halo around one of the nuclei. Fig. 16: apposed cells presenting a dilatation (observe a clearer region there), besides a large nucleus and a small stained body (arrow). Figs 17-18: note the peculiar chromatin arrangement in the bulb-like structure of these pairs.

taking into account that they had a constant pattern of features and were widespread in the cultures on a given occasion, furthermore seeming steps of a sequence.
Although with the data now at hand it was not possible to establish the dynamics of the phenomenon here described, after comparative examination of nearly 350 photomicrographs taken 

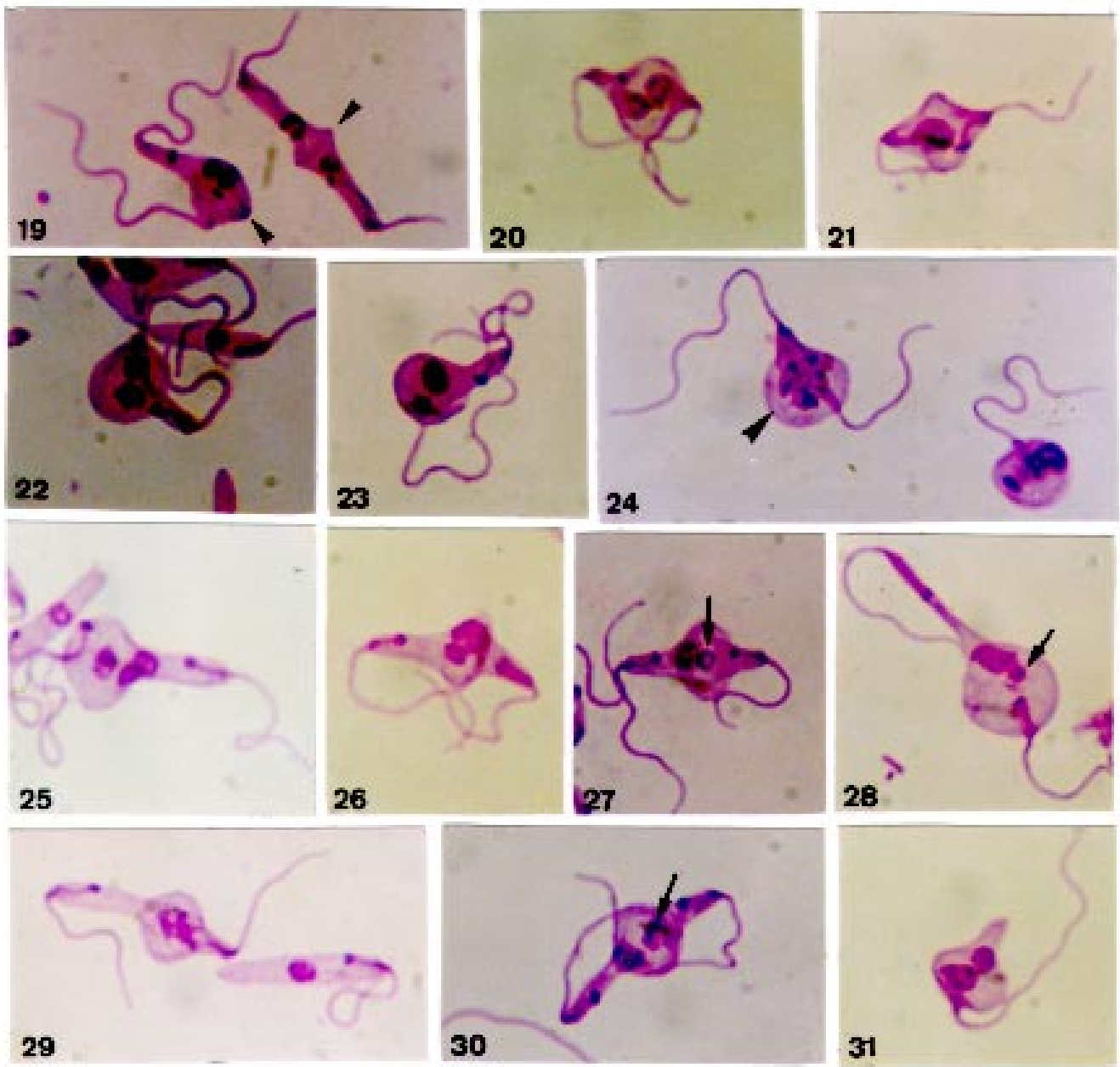

Unusual features in Phytomonas davidi cultured in LIT medium. Giemsa stain after $\mathrm{HCl}$ treatment. X 1,700. Fig. 19: different features of attached parasites; note the position of their nuclei, as well as the enlargement and the stained line at the cellular junction (arrowheads). Fig. 20: bulb-like structure holding two parasites, whose nuclei are placed there. Fig. 21: in this pair only one nucleus is seen. Figs 22-23: in these cases the bulb-like dilatation occupies entirely one parasite and almost wholly the other, their nucleus being there. Fig. 24: note the peculiar chromatin arrangement in the bulb-like structure of the joined cells (arrowhead), as well the monoflagellate round cell apparently with two nuclei very close each other. Fig. 25: pair of cells presenting the bulb-like dilatation in one of them and having three nuclei, two of which in close proximity. Fig. 26: this pair presents a large and a small nucleus near each other. Fig. 27-28: note the small stained body (arrow) beside the nucleus in the bulb-like structure, which is very well-developed in the pair shown in figure 28 , where also a slim cell appears almost detaching. Fig. 29: the nuclei of the joined cells are not individualized in this pair, whose chromatin appears weakly stained. Fig. 30: in the bulb-like dilatation one of the nuclei seems degenerating. Fig. 31: sucker-like cell with two nuclei.

on Giemsa-stained smears, some sequences of events are tentatively suggested, as follows. The putative fusion of two apposed promastigotes proceeds and at their junction appears a bulb-like dilatation (as in Fig. 4); the nucleus of each cell migrates towards the other, they usually fusing inside or near such dilatation (as suggested in Figs 4-6). In some instances, the bulb-like structure in- creases holding together both parasites (as in Figs $7-9,20$ ), thus arising biflagellate round cells with two nuclei or a single large one (as in Fig. 10). In other cases, only one parasite of the pair is occupied by the bulb-like structure, becoming gradually round (as in Figs 11, 13). Subsequently, the cells of such pair are separated releasing monoflagellate round forms with two nuclei or a 
single one (sometimes accompanied by a small stained body), besides flagellate slender cells without nucleus (as suggested in Figs 11-14). Although it is not easy to know the fate of the above-cited round cells, it seems that at least some of them can restore its original form (promastigote), as mainly suggested by several sucker-like cells found in the cultures (as in Fig. 31).

A peculiar structure found throughout this study was the small stained body, which sometimes occurred either in pairs of apposed parasites or in round cells, generally near a single large nucleus (Figs 13, 14, 16, 27, 28). Two possibilities have been considered to explain its occurrence: (1) it could be a residual chromatin after a putative fusion of two nuclei; (2) it would be one degenerating nucleus of a pair of joined cells, which previously presented either two or three nuclei. Figs 15 and 16 , as well as 25 and 26 , suggest that two nuclei can fuse, remaing the third one to degenerate; Fig. 30 suggests one nucleus in degeneration (arrowhead).

Whether the cytological findings reported in this paper can be ascribed to an actual sexuality in $P$. davidi, or simply represent an ancient behavior which is being gradually left off, with no genetic exchange occurring there, at present it is difficult to establish. However, it is noticeable that several of them display opportunities for fusion of two parasites and interactions between nuclei (as in Figs $5-11,17,18,21,24,29)$, thus fulfilling some prerequisites for sex and genetic exchange.

The phenomenon under study was infrequent in $P$. davidi cultures. After its discovery, it gradually disappeared, being seen again only after more than five years. Initially I had considered the possibility of its being triggered by a marked change in the parasite environment following the culture recovery from liquid nitrogen storage, as it was supplied. However, this could not be proved, since such phenomenon did not occur again after defrosting a culture cryopreserved to verify this hypothesis, as well as its reappearance took place in cultures which were not coming from cryopreservation. Thus, further investigation is needed not only to know the reason(s) of the appearance of this phenomenon in $P$. davidi, but also to better understand it by different approaches: at the ultrastructural level, using techniques of molecular genetics to assay the possibility of genetic exchange, as well as trying to dynamically record it.

\section{ACKNOWLEDGMENTS}

To Dr Wladimir Lobato Paraense for reviewing the manuscript and Dr Monika Barth for allowing the photomicrographs to be taken at her laboratory.

\section{REFERENCES}

Belli AA, Miles MA, Kelly JM 1994. A putative Leishmania panamensis/Leishmania braziliensis hybrid is a causative agent of human cutaneous leishmaniasis in Nicaragua. Parasitology 109: 435-442.

Camargo EP, Sbravate C, Teixeira MMG, Uliana SRB, Affonso MHT, Floeter-Winter LM 1992. Ribosomal DNA restriction analysis and synthetic oligonucleotide probing in the identification of genera of lower trypanosomatids. J Parasitol 78: 40-48.

Carvalho ALM 1973. Estudos sobre a posição sistemática, a biologia e a transmissão de tripanosomatídeos encontrados em Zelus leucogrammus (Perty, 1834) (Hemiptera, Reduviidae). Rev Pat Trop 2: 223-274.

Chiari E, Camargo EP 1984. Culturing and cloning of Trypanosoma cruzi, p. 23-26. In CM Morel, Genes and Antigens of Parasites. A Laboratory Manual, Fundação Oswaldo Cruz, Rio de Janeiro.

Cupolillo E, Grimaldi Jr G, Momen H 1992. Hybrid parasites in the Leishmania braziliensis "complex" causing cutaneous leishmaniasis in humans. Isozyme Bull 25: 51.

Degen R, Pospichal H, Enyaru J, Jenni L 1995. Sexual compatibility among Trypanosoma brucei isolates from an epidemic area in southeastern Uganda. Parasitol Res 81: 253-257.

França C 1920. La flagellose des Euphorbes. Ann Inst Pasteur 34: 432-435.

Gibson W, Bailey M 1994. Genetic exchange in Trypanosoma brucei: evidence for meiosis from analysis of a cross between drug-resistant transformants. $\mathrm{Mol}$ Biochem Parasitol 64: 241-252.

Glassberg J, Miyazaki L, Rifkin MR 1985. Isolation and partial characterization of mutants of the trypanosomatid Crithidia fasciculata and their use in detecting genetic recombination. J Protozool 32: 118-125.

Jenni L, Marti S, Schweizer J, Betschart B, Le Page RWF, Wells JM, Tait A, Paindavoine P, Pays E, Steinert M 1986. Hybrid formation between African trypanosomes during cyclical transmission. $\mathrm{Na}$ ture 322: 173-175.

Kelly JM, Law JM, Chapman CJ, Van Eys GJJM, Evans DA 1991. Evidence of genetic recombination in Leishmania. Mol Bioch Parasitol 46: 253-264.

Lanotte G, Rioux J-A 1990. Fusion cellulaire chez les Leishmania (Kinetoplastida, Trypanosomatidae). CR Acad Sci Paris 310 (série III): 285-288.

LeBlancq SM, Schnur LF, Schlein Y 1983. An apparent association of enzymic variants of Leishmania major with specific geographical areas in Brazil. Bull Soc Path Exot 76: 543-548.

Maazoun R, Lanotte G, Rioux J-A, Pasteur N, KillickKendrick R, Pratlong F 1981. Signification du polymorphisme enzymatique chez les leishmanies. Ann Parasitol Hum Comp 56: 467-475.

McGhee RB, Postell FJ 1976. Axenic cultivation of Phytomonas davidi Lafont (Trypanosomatidae), a symbiote of laticiferous plants (Euphorbiaceae). $J$ Protozool 23: 238-241. 
Sousa MA 1994. Cell-to-cell interactions suggesting a sexual process in Herpetomonas megaseliae (Kinetoplastida: Trypanosomatidae). Parasitol Res 80: 112-116.

Sousa MA, Côrte-Real S, Pereira MCS, Garcia L 1993. Leishmania major: cytological events suggesting sexuality. Mem Inst Oswaldo Cruz 88
(Suppl): 121.

Tait A, Turner CMR 1990. Genetic exchange in Trypanosoma brucei. Parasitol Today 6: 70-75.

Wallace FG, Roitman I, Camargo EP 1992. Trypanosomatids of plants, p. 55-84. In JP Kreier, JR Baker (eds), Parasitic Protozoa, vol. 2, 2nd ed. Academic Press, New York. 